\title{
Differentiating resting brain states using ordinal symbolic analysis
}

Carlos Quintero-Quiroz, Luis Montesano, Antonio J. Pons, M. C. Torrent, Jordi García-Ojalvo, and Cristina Masoller

Citation: Chaos 28, 106307 (2018); doi: 10.1063/1.5036959

View online: https://doi.org/10.1063/1.5036959

View Table of Contents: http://aip.scitation.org/toc/cha/28/10

Published by the American Institute of Physics

\section{Articles you may be interested in}

Complexity and irreducibility of dynamics on networks of networks

Chaos: An Interdisciplinary Journal of Nonlinear Science 28, 106306 (2018); 10.1063/1.5039483

Statistics of inverse interspike intervals: The instantaneous firing rate revisited

Chaos: An Interdisciplinary Journal of Nonlinear Science 28, 106305 (2018); 10.1063/1.5036831

Neuron dynamics variability and anomalous phase synchronization of neural networks

Chaos: An Interdisciplinary Journal of Nonlinear Science 28, 106304 (2018); 10.1063/1.5023878

Propagation delays determine neuronal activity and synaptic connectivity patterns emerging in plastic neuronal networks

Chaos: An Interdisciplinary Journal of Nonlinear Science 28, 106308 (2018); 10.1063/1.5037309

Efficient determination of synchronization domains from observations of asynchronous dynamics

Chaos: An Interdisciplinary Journal of Nonlinear Science 28, 106301 (2018); 10.1063/1.5037012

Harnessing stochasticity: How do organisms make choices?

Chaos: An Interdisciplinary Journal of Nonlinear Science 28, 106309 (2018); 10.1063/1.5039668

\section{Don't let your writing keep you from getting published!}

\section{AIP Author Services}




\title{
Differentiating resting brain states using ordinal symbolic analysis
}

\author{
Carlos Quintero-Quiroz, ${ }^{1}$ Luis Montesano, ${ }^{2}$ Antonio J. Pons, ${ }^{3}$ M. C. Torrent, ${ }^{3}$ \\ Jordi García-Ojalvo, ${ }^{4}$ and Cristina Masoller ${ }^{3}$ \\ ${ }^{1}$ Departament de Física, Universitat Politècnica de Catalunya, Colom 11, 08222 Terrassa, Barcelona, Spain \\ ${ }^{2}$ Bitbrain, Paseo de Sagasta, 19, 50008 Zaragoza, Spain \\ ${ }^{3}$ Departament de Física, Universitat Politècnica de Catalunya, Rambla St. Nebridi 22, 08222 Terrassa, \\ Barcelona, Spain \\ ${ }^{4}$ Department of Experimental and Health Sciences, Universitat Pompeu Fabra, Parc de Recerca Biomèdica de \\ Barcelona, Carrer del Dr. Aiguader, 88, 08003 Barcelona, Spain
}

(Received 19 April 2018; accepted 25 July 2018; published online 8 October 2018)

\begin{abstract}
Symbolic methods of analysis are valuable tools for investigating complex time-dependent signals. In particular, the ordinal method defines sequences of symbols according to the ordering in which values appear in a time series. This method has been shown to yield useful information, even when applied to signals with large noise contamination. Here, we use ordinal analysis to investigate the transition between eyes closed (EC) and eyes open (EO) resting states. We analyze two electroencephalography datasets (with 71 and 109 healthy subjects) with different recording conditions (sampling rates and the number of electrodes in the scalp). Using as diagnostic tools the permutation entropy, the entropy computed from symbolic transition probabilities, and an asymmetry coefficient (that measures the asymmetry of the likelihood of the transitions between symbols), we show that the ordinal analysis applied to the raw data distinguishes the two brain states. In both datasets, we find that, during the EC-EO transition, the EO state is characterized by higher entropies and lower asymmetry coefficient, as compared to the EC state. Our results thus show that these diagnostic tools have the potential for detecting and characterizing changes in time-evolving brain states. Published by AIP Publishing. https://doi.org/10.1063/1.5036959
\end{abstract}

In the "big data" era, many efforts are being devoted to extracting useful information from complex signals. The human brain is one of the most complex systems that one can try to understand. In the last few decades, the development and popularization of recording techniques, such as electroencephalography (EEG), magnetoencephalography (MEG), and functional magnetic resonance imaging (fMRI), have provided the scientific community with a huge amount of data: different types of brain signals, recorded with different spatio-temporal resolution, under different behavioral or cognitive states, from healthy or from dysfunctioning subjects. The underlying brain states are, in spite of many efforts, still poorly understood. Here, we use a symbolic analysis tool to investigate EEG signals recorded from healthy subjects during a simple behavioral task: the subjects remain in the resting state with eyes closed (EC state) during an interval of time and then open their eyes (EO state). We show that symbolic analysis applied to the raw EEG signals detects the transition and identifies subtle differences between the $\mathrm{EC}$ and $\mathrm{EO}$ brain states.

\section{INTRODUCTION}

Changes in brain states detected through the analysis of electroencephalography (EEG) signals can be used for translating brain signals into operational commands, and in fact, EEG analysis is one of the techniques used for brain-computer interfaces.
Several methods have been used to detect underlying changes in the behavior of dynamical systems from observed data, and one of these, ordinal analysis, ${ }^{1-3}$ has been demonstrated to be computationally efficient and to perform well even with very noisy data. ${ }^{4,5}$ Due to these advantages, ordinal analysis has been used in neuroscience, ${ }^{6}$ for example, for studying epilepsy. ${ }^{7-13}$

Since the early 1930s, it is well known that alpha waves dominate the EEGs of healthy individuals when they are resting with their eyes closed and that this activity diminishes when their eyes are opened. ${ }^{14-17}$ Therefore, a simple method to detect the Eyes-Closed (EC) to Eyes-Open (EO) transition is by using the Fourier spectrum to estimate the difference of the power of the alpha frequency components. ${ }^{18,19}$ However, this approach has the drawback of requiring a certain time-window for computing the power spectra. Another approach to studying the EC-EO transition is to use the synchronization likelihood ${ }^{20}$ or the mutual information ${ }^{21}$ to find changes in the functional brain networks that characterize the two brain states. However, constructing functional brain networks is computationally demanding, and comparing them is a challenging task because it is not always possible to discriminate reliably between differences that are due to constraints imposed by the method of network construction or due to genuine changes in brain states. $^{22,23}$

The aim of this paper is to investigate if the ordinal approach can accurately discriminate between EC and EO brain states. In Sec. II, we describe the datasets analyzed; in Sec. III, we describe the ordinal-pattern methodology and 
TABLE I. Description of the datasets used.

\begin{tabular}{lcc}
\hline \hline & DTS1 & DTS2 \\
\hline Sampling rate (Hz) & 256 & 160 \\
Time task (seg) & 120 & 60 \\
Total points & 30720 & 9600 \\
Number of electrodes & 16 & 64 \\
Number of subjects & 71 & 109 \\
\hline \hline
\end{tabular}

the quantifiers used to characterize the EC and EO states. Section IV presents the results obtained, and Sec. V summarizes our conclusions.

\section{DATASETS}

We use two EEG datasets with a different number of subjects and recording conditions, which are summarized in Table I. Dataset one (DTS1) was collected by the Bitbrain company. ${ }^{24}$ The EEG signals were recorded from 71 healthy subjects that remained with eyes closed and eyes open during a period of two minutes each. Dataset two (DTS2), which is freely available, ${ }^{25,26}$ consists of EEG recordings of 109 subjects performing the same task, in this case for a period of one minute in each of the two states. For more information about the experimental recording setup (type of electrodes, cutting frequencies, etc.) and subjects (ages range, sex, etc.), refer to Refs. 24-26.

We removed the artifacts related to eye blinking following the standard procedure: we applied the Independent Component Analysis (ICA) using the function ICA from the MNE library on Python ${ }^{27,28}$ and filtered out the independent component related to the blinks (see Fig. 1).

The results of EEG spectral analysis are often grouped into the traditional frequency bands: $\delta \in(0.5,4] \mathrm{Hz}, \theta \in$ $(4,8] \mathrm{Hz}, \alpha \in(8,14] \mathrm{Hz}, \beta \in(14,30] \mathrm{Hz}$, and $\gamma \geq 30 \mathrm{~Hz}$. In fact, there is a lot of physiological and statistical evidence for the independence of several of these bands, but their limits may vary according to the particular experiment being considered, and they can be adjusted as necessary. It is well known that alpha waves are a dominant component in EEG signals during eyes closed conditions and are reduced when the eyes are open. ${ }^{17,18}$ This effect is observed more strongly in the occipital electrodes, mainly due to brain electrical activity from the primary visual cortex. Therefore, in order to determine whether changes detected through ordinal analysis are only due to the change of the strength of alpha waves, we analyze and compare the results obtained from the raw time series and from the post-processed time series where both eye blinking artifacts and the frequency component of the alpha band were removed (and focusing in the alpha band by using a bandpass filter between 14 and $31 \mathrm{~Hz}$, see Fig. 2).

\section{METHODS}

We apply ordinal analysis in non-overlapping windows of $1 \mathrm{~s},{ }^{8}$ and thus, the number of data points in the window, $w$, is equal to 256 for DTS1 and to 160 for DTS2. Then, for each electrode $i$, the time-series, $x_{i}(t)=\{x(1), x(2), \ldots, x(w)\}$, is transformed into a sequence of symbols, $s_{i}(t)$, by using the ordinal rule, ${ }^{1,4}$ explained in what follows.

To define the ordinal patterns, we consider vectors of dimension $D$ formed by consecutive data points, i.e., $\{x(j), x(j+1), \ldots, x(j+D-1)\}$, and then assign a symbol according to the ordinal relationship (from the largest to the smallest value) of the $D$ entries in the vectors. For example, with $D=2$, there are 2 ordinal patterns: $(D !): x\left(t_{j}\right)<x\left(t_{j+1}\right)$ corresponding to the ordinal pattern " 01 " and $x\left(t_{i}\right)>x\left(t_{i+1}\right)$ corresponding to the ordinal pattern " 10 ." Then, we compute the frequency of the occurrence of the D! different patterns in the signal of electrode $i$ and with it, evaluate the corresponding permutation entropy (PE) by

$$
\mathrm{PE}^{i}=-\sum_{k}^{D !} p_{\pi_{k}} \ln p_{\pi_{k}},
$$

where $p_{\pi_{k}}$ is the probability of pattern $\pi_{k}$.

Then, the average over all the electrodes

$$
\langle\mathrm{PE}\rangle=\frac{1}{N \text { [electrodes }]} \sum_{i} \mathrm{PE}^{i}
$$

is taken, with this average the $\langle\mathrm{PE}\rangle$ is a measure of the entropy of the brain EEG signals, in the given time window. If the EEG signals are generated by fully random processes, all symbols are equally probable and the PE is maximum, $\mathrm{PE}=\ln (D !)$.
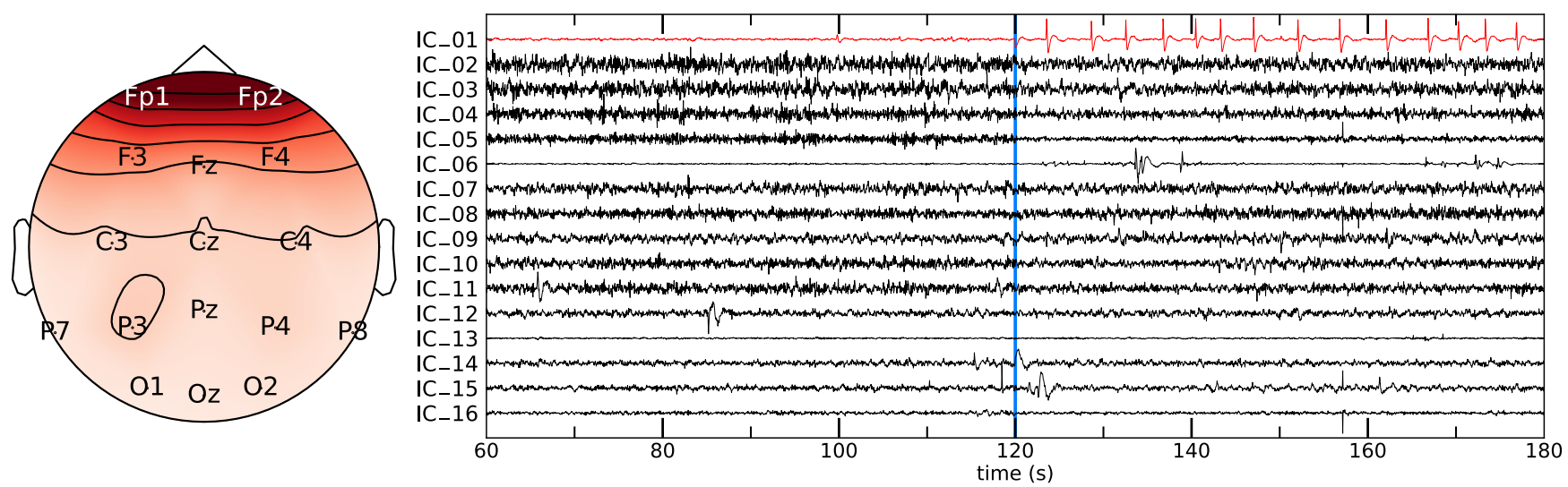

FIG. 1. Example of Independent Components (IC) for a given subject of DTS1 for eye blink removal. Left: spatial representation of the mixing vector for the eye blinking component (IC_01 plotted in red in right panel). Right: time course of all the independent components obtained from the ICA function. 

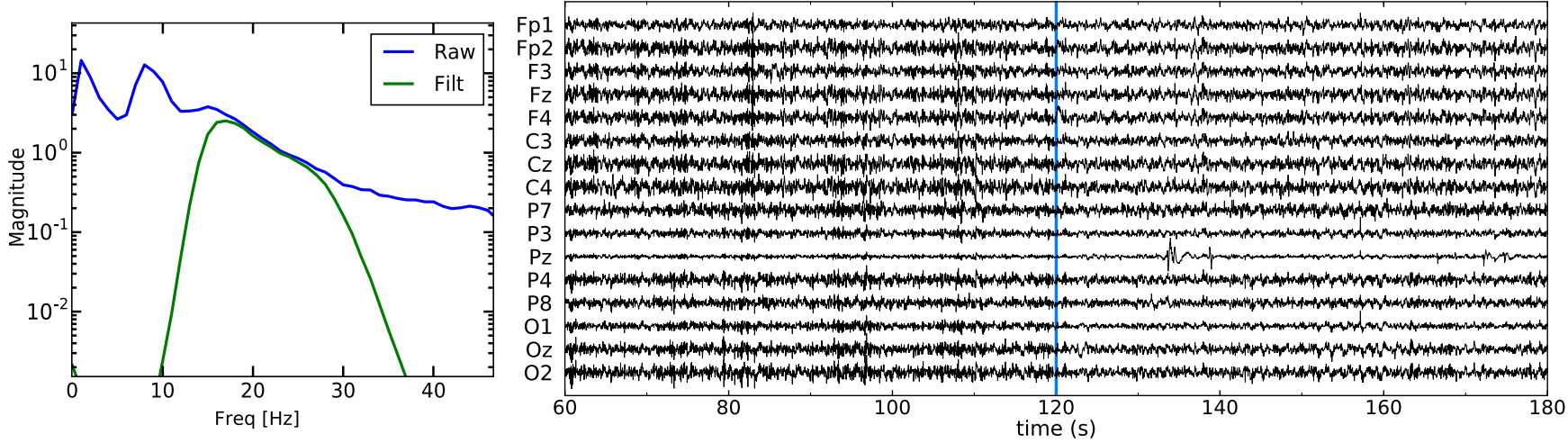

FIG. 2. Post-processed data, artifacts removed using the ICA method and bandpass filter in the beta band to remove the alpha band component. The left panel displays the power spectrum of the EEG of a subject before (blue line) and after (green line) filtering. The right panel displays the post-processed EEG's time-series; the vertical line indicates the time of the eyes closed-eyes open transition. The subject is the same as in Fig. 1.

Additional diagnostic tools were proposed by Masoller et al., ${ }^{3}$ which are based in the transition probabilities (TPs) between consecutive symbols defined from non-overlapping data values. The transition probability from pattern $\pi_{a}$ to pattern $\pi_{b}$ is the relative number of times pattern $\pi_{a}$ is followed by pattern $\pi_{b}$, in the sequence $s(t)$ :

$$
M_{a, b}=\frac{\sum_{t}^{w-1} N\left[s(t)=\pi_{a}, s(t+1)=\pi_{b}\right]}{\sum_{t}^{w-1} N\left[s(t)=\pi_{a}\right]} .
$$

With this definition, the transition probabilities are normalized such that $\sum_{b} M_{a b}=1$. Then, exploiting this normalization, an entropy can be associated to the transition probabilities of each pattern as $s_{a}=-\sum_{b} M_{a b} \ln M_{a b}$, and its average

$$
s_{n}=\frac{\sum_{a} s_{a}}{D !}
$$
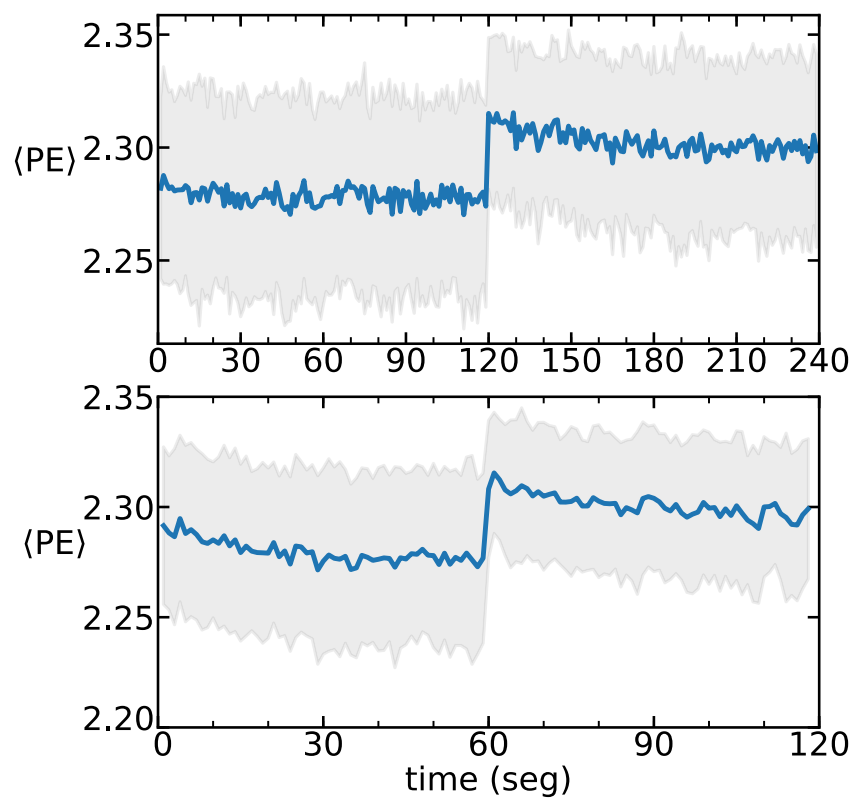

FIG. 3. Permutation entropy, Eq. (2), from a raw time series of DTS1 (top) and DTS2 (bottom). In DTS1, the subjects open their eyes at $120 \mathrm{~s}$; in DTS2, at $60 \mathrm{~s}$. The blue line indicates the mean value of the $\langle\mathrm{PE}\rangle$ for all the subjects, and the shaded area indicates one standard deviation of the $\langle\mathrm{PE}\rangle$ values. is another measure of the entropy of the EEG signal. If a signal is generated by fully random processes, all transition probabilities will be equal, and thus, $s_{a}=\ln (D$ !) for all $\pi_{a}$, and $s_{n}=\ln (D !)$. In addition, we calculate the transition asymmetry coefficient ${ }^{3}$

$$
a_{c}=\frac{\sum_{a} \sum_{b \neq a}\left|M_{a b}-M_{b a}\right|}{\sum_{a} \sum_{b \neq a}\left(M_{a b}+M_{b a}\right)},
$$

which is equal to zero if transition probabilities are fully symmetric $\left(M_{a b}=M_{b a}\right.$ for all $\left.\pi_{a}, \pi_{b}\right)$ and equal to one if they are fully asymmetric (either $M_{a b}=0$ or $M_{b a}=0$ for all $\pi_{a}, \pi_{b}$ ). If the EEG signals are generated by fully random processes, then the transition probabilities will be all equal and $a_{c}=0$.

In Sec. IV, the analysis is performed with non-overlapping patterns of length $D=4$ (similar results were found with $D=3$ ). There are $4 !=24$ possible patterns and $24 \times 24=$ 576 possible transitions. For the dataset DTS1 (DTS2), taking together the 16 (64) electrodes, in each time window the symbolic sequence contains 4048 (10048) patterns and 4032
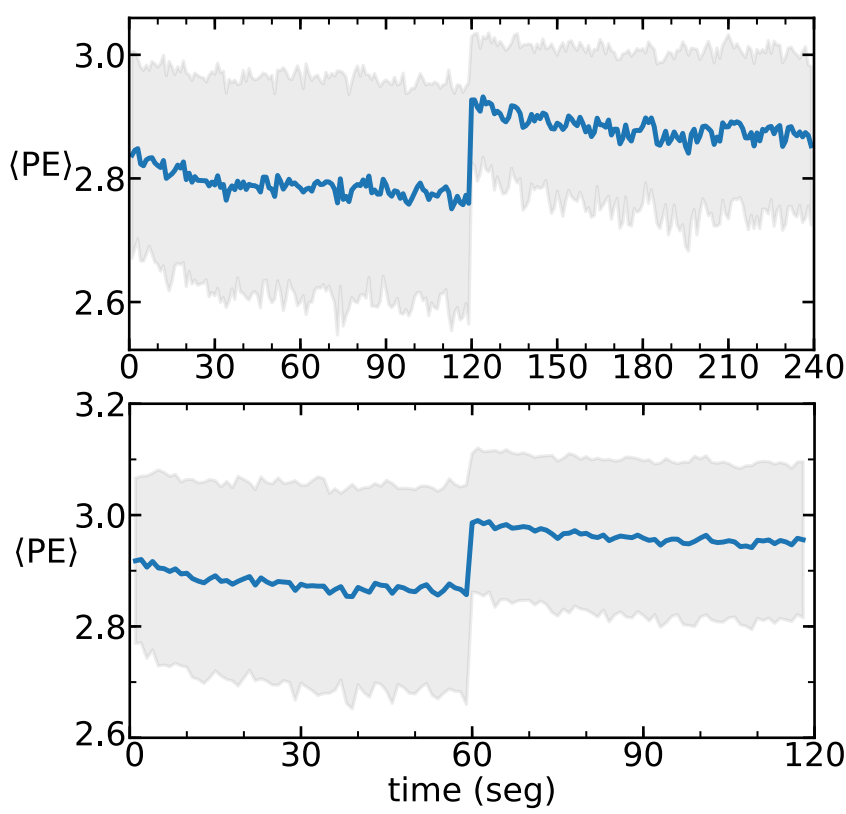

FIG. 4. Permutation entropy, Eq. (2), computed from post-processed time series of DTS1 (top) and DTS2 (bottom). 


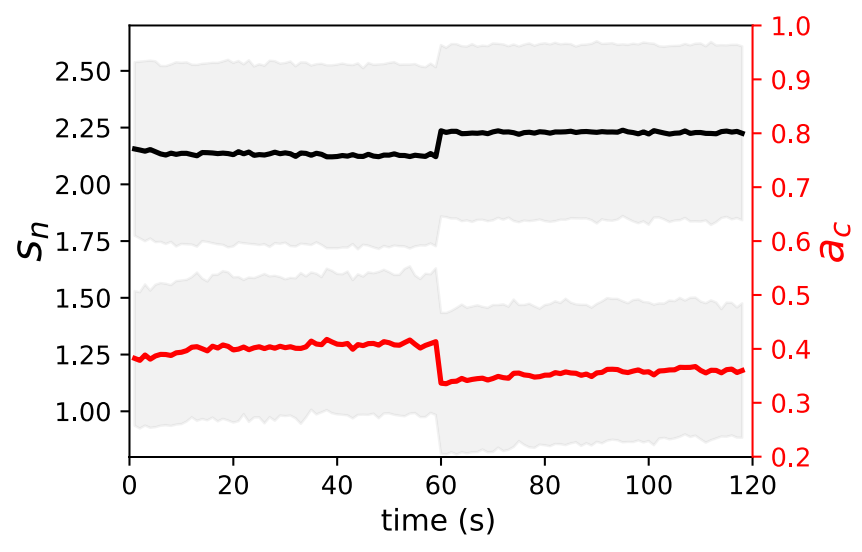

FIG. 5. The entropy defined from the transition probabilities, Eq. (4), and the asymmetry coefficient, Eq. (5), computed for the DTS2.

(9984) transitions. While the number of patterns is clearly sufficient to compute the probabilities of the 24 patterns with good accuracy, longer sequences are needed to compute the 576 TPs with similar accuracy. Nevertheless, we will show that the TP-based diagnostic tools, $s$ and $a_{c}$, can also detect changes in datasets. As a measure of statistical significance, we calculate the $p$-value using Welch's t-test and consider, as null hypothesis, that the signals represent the same state.

\section{RESULTS}

We begin by calculating the $\langle\mathrm{PE}\rangle$ for the raw, preprocessed time series. Figure 3 displays the results obtained from DTS1 and DTS2 and we can see that there is a significant difference between the $\langle\mathrm{PE}\rangle$ values of the eyes-closed and eyes-open states. The entropy is computed for each subject and then is averaged over all the subjects ( 71 or 109 , depending on the dataset). The shaded area represents one standard deviation of the $\langle\mathrm{PE}\rangle$ values of all subjects, and we note that there is large variability; thus, the $\langle\mathrm{PE}\rangle$ value does not allow for a full discrimination between the two states. We note that the average $\langle\mathrm{PE}\rangle$ value is slightly different for the two datasets, which is attributed to the fact that they have different spatial and temporal resolution. We also note that the average value of the $\langle\mathrm{PE}\rangle$ is significantly different from the maximum possible value (which occurs when the patterns are equally probable, and for $D=4, \mathrm{PE}_{\max }=\ln 24=3.18$ ). This reveals the presence of patterns with high and low frequency of occurrence in the symbolic sequence. An inspection of the ordinal probabilities reveals that the "trend" patterns (generated by consecutively increasing or decreasing data values) are more expressed, in all the channels and for both EO and EC states. For future work, it would be interesting to investigate if the probabilities of certain patterns (which could be defined by using a lag, as in Ref. 29) allow for a better discrimination of the two states.

Comparing with the results obtained from the postprocessed time series, displayed in Fig. 4, we note that the $\langle\mathrm{PE}\rangle$ behavior remains almost unchanged, which suggests that the $\langle\mathrm{PE}\rangle$ captures changes in brain dynamics which are not due to the change in the strength of alpha oscillations during the EC-EO transition.

Figure 5 displays the TP-based measures, $s$ and $a_{c}$, using the DST2 (similar results were found in DST1), although there is a clear transition around $60 \mathrm{~s}$ in the mean values, the dispersion in the values of the different subjects is higher than in the $\langle\mathrm{PE}\rangle$ analysis (likely due to the limited length of the time series, which does not allow a precise estimation of the TPs).

In Figs. 6 (for DTS1) and 7 (for DTS2), we present the topographic visualization, for the different electrodes, of the $\mathrm{PE}$ value averaged over all the subjects, for the EC and EO
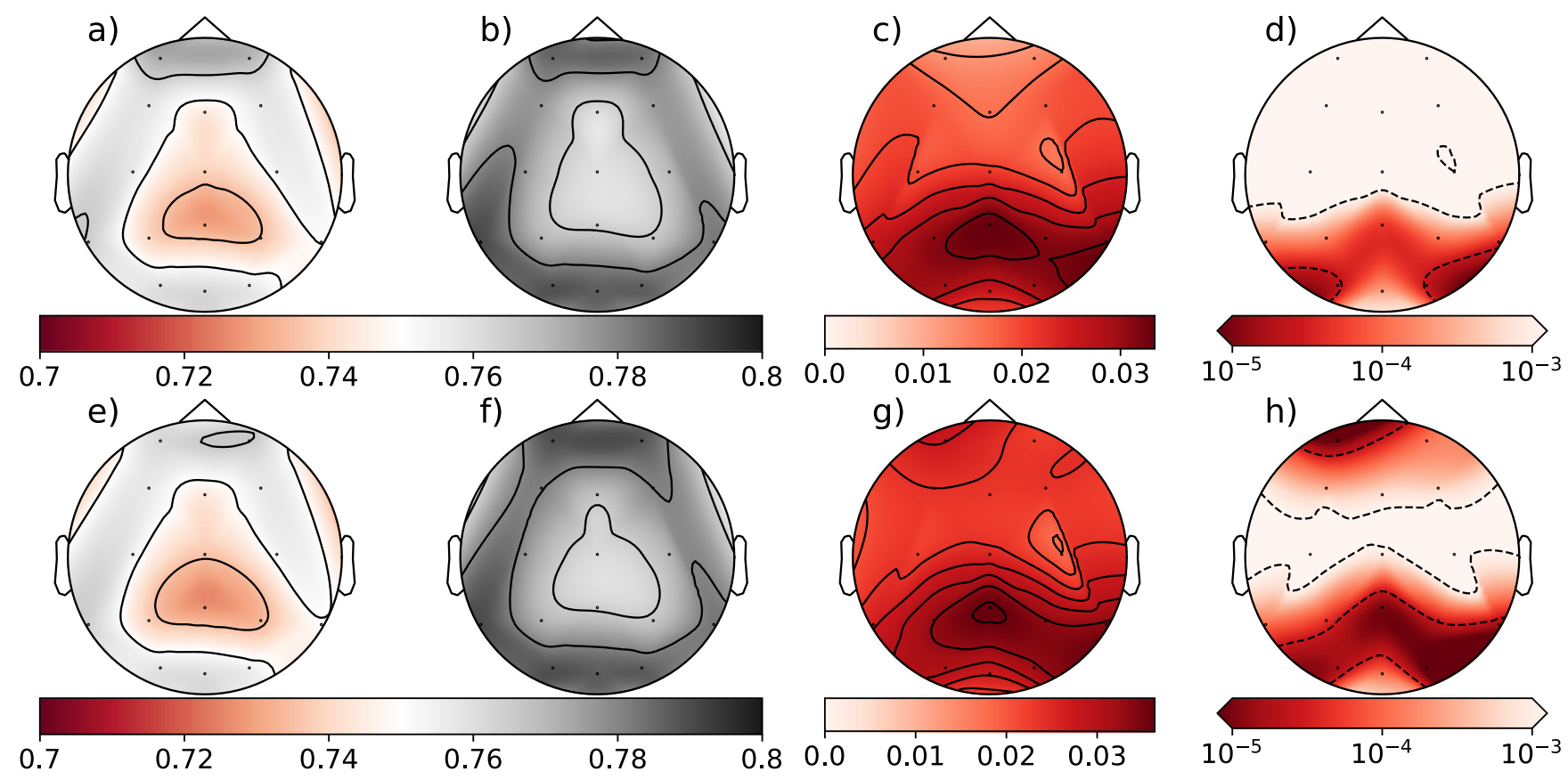

FIG. 6. Topographic visualization of the analysis of the raw (top row) and post-processed (lower row) EEG signals of DTS1, average over the subjects. Panels (a) and (e) display the normalized permutation entropy $[\langle\mathrm{PE}\rangle / \ln (D !)]$ for EC conditions; (b) and (f) for EO conditions; (c) and (g) display the difference of the $\langle\mathrm{PE}\rangle$ values; (d) and (h) display the $p$ value. 

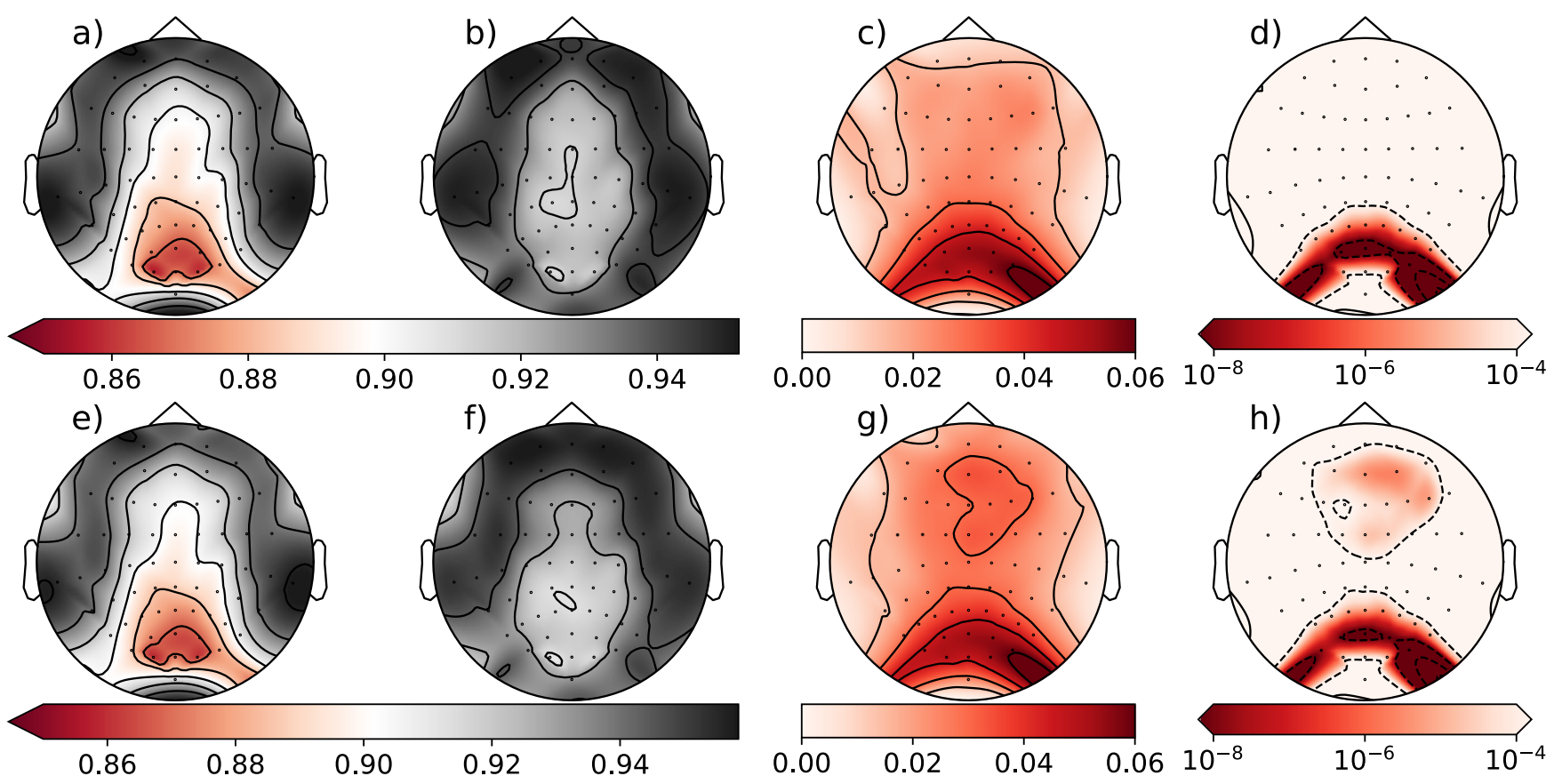

FIG. 7. As in Fig. 6, but for the dataset DTS2.

conditions. We also present the difference of PE values (PEopen-PE-closed) and the $p$-value. The results are consistent for the two datasets; the discrepancies are due to their different spatial resolution. We also note the low p-values are obtained, which confirm the significance of the uncovered differences.

\section{CONCLUSIONS}

We have used ordinal time series analysis to investigate EEG signals recorded under eyes-closed (EC) and eyes-open (EO) resting conditions. We have analyzed two datasets with different spatial and temporal resolutions and contrasted the results of the analysis of raw time series and post-processed time series (where eye blinking artifacts were removed and the alpha frequency band was filtered out). We used three diagnostic measures, the permutation entropy, $\langle\mathrm{PE}\rangle$, which is computed from the probabilities of the ordinal patterns, and two measures, the transition entropy and the asymmetry coefficient, which are computed from the transition probabilities between patterns.

During the EC-EO transition, we have found, in both datasets, that the EO state is characterized by higher entropy values, accompanied by a lower asymmetry coefficient, with respect to the EC state. Considering the analysis of Barry et $a .^{18}$ of the EC-EO-EC transitions, it is not impossible to discard that the detected significant variations are relative to the EC-EO transition (and not absolute characteristic values of the EC and EO states). We have also identified which brain regions are more important for distinguishing the two states during the EC-EO transition. No significant difference was detected between the raw data and the postprocessed data, which suggests that the ordinal method can be directly applied to EEG signals, avoiding the need of data post-processed. Nonetheless, ordinal analysis can be a computationally efficient tool, which could provide extra valuable information for new brain-computer interface protocols.

It is well known that EEG is a method that depends on contingent factors such as air humidity, skin conductance, electrode resistance, and arousal, among others. These factors may change to a large extent among individuals at the time of recording the EEG. Therefore, our results might be due to a sub-sample of individuals overemphasizing the jump at the transition, whereas other individuals show little significant changes in the measured variables. However, we remark that we obtain qualitatively the same results in the two databases. Therefore, we speculate that environmental conditions, individual characteristics, etc., do not influence the ordinal pattern statistics. Because ordinal analysis is robust to noise, the raw data and the filtered data give the same results.

\section{ACKNOWLEDGMENTS}

This work was supported in part by ITN NETT (FP7 289146), the Spanish MINECO (FIS2015-66503 and FIS2015-66503-C3-2-P), and the program ICREA ACADEMIA of Generalitat de Catalunya.

${ }^{1}$ C. Bandt and B. Pompe, Phys. Rev. Lett. 88, 174102 (2002).

${ }^{2}$ Y. Cao, W.-w. Tung, J. Gao, V. A. Protopopescu, and L. M. Hively, Phys. Rev. E 70, 046217 (2004).

${ }^{3}$ C. Masoller, Y. Hong, S. Ayad, F. Gustave, S. Barland, A. J. Pons, S. Gómez, and A. Arenas, New J. Phys. 17, 023068 (2015).

${ }^{4}$ M. Zanin, L. Zunino, O. A. Rosso, and D. Papo, Entropy 14, 1553 (2012).

${ }^{5}$ C. Quintero-Quiroz, S. Pigolotti, M. C. Torrent, and C. Masoller, New J. Phys. 17, 093002 (2015).

${ }^{6}$ C. A. Stefano Filho, R. Attux, and G. Castellano, PeerJ 5, e3983 (2017).

${ }^{7}$ J. Li, J. Yan, X. Liu, and G. Ouyang, Entropy 16, 3049 (2014).

${ }^{8}$ N. Nicolaou and J. Georgiou, Expert Syst. Appl. 39, 202 (2012).

${ }^{9}$ E. Olofsen, J. Sleigh, and A. Dahan, Br. J. Anaesth. 101, 810 (2008).

${ }^{10}$ A. A. Bruzzo, B. Gesierich, M. Santi, C. A. Tassinari, N. Birbaumer, and G. Rubboli, Neurol. Sci. 29, 3 (2008). 
${ }^{11}$ X. Li, G. Ouyang, and D. A. Richards, Epilepsy Res. 77, 70 (2007).

${ }^{12}$ I. Veisi, N. Pariz, and A. Karimpour, in Proceedings of the Seventh IEEE International Conference on Bioinformatics and Bioengineering, 2007 (IEEE, 2007), pp. 200-203.

${ }^{13}$ X. Ren, Q. Yu, B. Chen, N. Zheng, and P. Ren, in 2015 20th Asia and South Pacific Design Automation Conference (ASP-DAC) (IEEE, 2015), pp. 20-21.

${ }^{14}$ J. R. Smith, Pedagog. Semin. J. Genet. Psychol. 53, 455 (1938).

${ }^{15}$ H. H. Jasper, Science 83, 259 (1936).

${ }^{16}$ E. D. Adrian and B. H. Matthews, Brain 57, 355 (1934).

${ }^{17}$ H. Berger, Arch. Psychiatr. Nervenkr. 98, 231 (1933).

${ }^{18}$ R. J. Barry, A. R. Clarke, S. J. Johnstone, C. A. Magee, and J. A. Rushby, Clin. Neurophysiol. 118, 2765 (2007).

${ }^{19}$ R. J. Barry and F. M. De Blasio, Biol. Psychol. 129, 293 (2017).

${ }^{20}$ S.-H. Jin, W. Jeong, D.-S. Lee, B. S. Jeon, and C. K. Chung, J. Neurophysiol. 111, 1455 (2014).

${ }^{21}$ B. Tan, X. Kong, P. Yang, Z. Jin, and L. Li, Comput. Math. Methods Med. 2013, 976365 (2013)
${ }^{22}$ B. C. Van Wijk, C. J. Stam, and A. Daffertshofer, PLoS One 5, e13701 (2010).

${ }^{23}$ T. A. Schieber, L. Carpi, A. Díaz-Guilera, P. M. Pardalos, C. Masoller, and M. G. Ravetti, Nat. Commun. 8, 13928 (2017).

${ }^{24}$ See http://bitbrain.tech/ for Bitbrain Technologies.

${ }^{25}$ G. Schalk, D. J. McFarland, T. Hinterberger, N. Birbaumer, and J. R. Wolpaw, IEEE Trans. Biomed. Eng. 51, 1034 (2004).

${ }^{26}$ A. L. Goldberger, L. A. Amaral, L. Glass, J. M. Hausdorff, P. C. Ivanov, R. G. Mark, J. E. Mietus, G. B. Moody, C.-K. Peng, and H. E. Stanley, Circulation 101, e215 (2000).

${ }^{27}$ A. Gramfort, M. Luessi, E. Larson, D. A. Engemann, D. Strohmeier, C. Brodbeck, L. Parkkonen, and M. S. Hämäläinen, Neuroimage 86, 446 (2014).

${ }^{28}$ A. Gramfort, M. Luessi, E. Larson, D. A. Engemann, D. Strohmeier, C. Brodbeck, R. Goj, M. Jas, T. Brooks, L. Parkkonen et al., Front. Neurosci. 7, 267 (2013).

${ }^{29}$ U. Parlitz, S. Berg, S. Luther, A. Schirdewan, J. Kurths, and N. Wessel, Comput. Biol. Med. 42, 319 (2012). 